\title{
Interleukin-8 in Hyperlipidemia and Coronary Heart Disease in Thai Patients Taking Statin Cholesterol-Lowering Medication While Undergoing Coronary Artery Bypass Grafting Treatment
}

\author{
Wilanee Dechkhajorn, ${ }^{1}$ Yaowapa Maneerat $\left(\mathbb{D},{ }^{1}\right.$ Kriengchai Prasongsukarn, ${ }^{2}$ \\ Panan Kanchanaphum $\mathbb{D}^{3},{ }^{3}$ and Ratchanok Kumsiri $\mathbb{D}^{3}$ \\ ${ }^{1}$ Department of Tropical Pathology, Faculty of Tropical Medicine, Mahidol University, Bangkok 10400, Thailand \\ ${ }^{2}$ Phramongkutklao Hospital, Bangkok 10400, Thailand \\ ${ }^{3}$ Department of Medical Science, Faculty of Science, Rangsit University, Pathumthani 12000, Thailand
}

Correspondence should be addressed to Ratchanok Kumsiri; ratchanok.k@rsu.ac.th

Received 11 March 2020; Revised 20 May 2020; Accepted 25 May 2020; Published 25 June 2020

Academic Editor: Leila Beltrami Moreira

Copyright (C) 2020 Wilanee Dechkhajorn et al. This is an open access article distributed under the Creative Commons Attribution License, which permits unrestricted use, distribution, and reproduction in any medium, provided the original work is properly cited.

\begin{abstract}
The role of interleukin-8 (IL-8), a pivotal chemokine in atherogenesis and coronary heart disease (CHD) development, is diverse and remains unclear. This cross-sectional study investigates the association of the IL- 8 expression in hyperlipidemia (H) and CHD patients who have been treated with statin cholesterol-lowering drugs while undergoing coronary artery bypass grafting treatment. Fifty-five Thai volunteers including 13 normal $(\mathrm{N}), 24 \mathrm{H}$, and $18 \mathrm{CHD}$ patients were enrolled for the investigation. All the CHD patients had been treated continuously with statin cholesterol-lowering medications since the disease was diagnosed and were undergoing coronary bypass grafting approximately one month later. Therefore, the CHD group was representative of a pathogenesis improvement in CHD. The IL8 mRNA expression was determined by real-time quantitative PCR in the peripheral blood mononuclear cells from heparinized blood. The plasma IL-8 levels were assessed by enzyme-linked immunosorbent assay. The result shows that the IL 8 mRNA expression in the H group tended to increase; however, in the CHD group, there was a significant decrease $(p=0.0111)$ compared to the N group. The IL8 mRNA expression and the plasma levels in the CHD group were significantly lower than those in the $\mathrm{H}$ group $(p<0.05)$. A significant negative correlation between the IL8 mRNA $(r=-0.499)$ or plasma IL-8 $(r=-0.3875)$ expression and CHD progression was observed $(p<0.05)$. In conclusion, the transcriptomic and the phenotypic IL-8 expression decreased significantly in the Thai CHD patients who had continuously received statin-group medications compared to the $\mathrm{H}$ and $\mathrm{N}$ group participants. Therefore, IL- 8 should serve as a feasible marker and could be used to evaluate the therapeutic effects of statins and illustrate the pathology of CHD treatment.
\end{abstract}

\section{Introduction}

Coronary heart disease (CHD) is one of the most significant vascular disorders in the world that is caused primarily by atherosclerosis complications. Major atherosclerosis disease mechanisms have been correlated to a high level of cholesterol, genetics, and the environment; however, the pathogenesis is still not understood entirely. Without treatment, atherosclerosis enhances occlusion of the arteries leading to ischemic heart disease and sudden myocardial infarction (MI) [1].
Extensive studies have investigated the role of inflammation in atherogenesis and coronary artery disease. The interplay of various inflammatory mediators, complements, and cytokines is involved in the pathogenesis of various stages of atherosclerosis from its initiation to progression, resulting in plaque formation and ruptures $[2,3]$. These inflammatory molecules include interferon (IFN), colonystimulating factors, interleukin (IL), chemokines, transforming growth factors (TGFs), and tumor necrosis factors (TNFs) (reviewed in $[2,4]$ ). The proinflammatory cytokine IFN- $\gamma$ can induce foam cell formation by increasing 
cholesterol retention through attenuating the expression of the ATP-binding cassette, subfamily A, as well as inducing the expression of acyl-CoA acyltransferase 1 that is involved in cholesterol esterification [4]. IL- $1 \alpha$, IL- $1 \beta$, and IL-18 are proinflammatory molecules involved in atherosclerosis disease progression [5], while the anti-inflammatory cytokines TGF- $\beta$, IL-33, IL-10, and IL-13 function in the reduction of atherogenesis in cardiovascular disorders $[4,6]$.

IL-8 is a proinflammatory cytokine or chemokine (CXCL8) produced by various cell types including endothelial cells, peripheral blood monocytes, and vascular smooth muscle cells. IL-8 is encoded by IL8 or CXCL8 [7]. Prior research on patients and cell culture models $[8,9]$ has shown that IL-8 is involved in the pathogenesis of cardiovascular disorders including CHD [10], MI [11], strokes, [12], and other diseases such as ankylosing spondylitis [13], emphysema [14], periodontitis [15], and systemic lupus erythematosus [16]. In CVD, IL-8 participates in all stages of atherosclerosis and the development of CHD [17-20].

Although numerous studies have reported the atherogenic effect and the association of increased IL-8 expression with the development of cardiovascular disorders and CHD, the pivotal roles of IL- 8 are still controversial. The antiatherogenic effect of IL-8 on myocardial tissue repair was documented in MI [21-23], while IL-8 serum levels were correlated with a decrease in the occurrence of MI among women [11]. Consistent with this, prior research using monkeys as models showed that atherosclerotic plaques in carotid bifurcations were mildly inversely correlated with plasma IL-8 levels [24].

In this cross-sectional study, we investigated the association of IL8 mRNA expression and the plasma IL-8 levels among healthy controls $(\mathrm{N})$, hyperlipidemia patients $(\mathrm{H})$, and CHD patients taking statin cholesterol-lowering medications while undergoing coronary bypass grafting in the CHD pathogenesis.

\section{Materials and Methods}

2.1. Materials. D-PBS (Wisent Inc., Quebec, Canada), TRIzol $^{\circledR}$ Reagent (Invitrogen ${ }^{\mathrm{TM}}$, Carlsbad, CA, USA), IsoPrep (Robbins Scientific Corporation, Sunnyvale, CA, USA), and the RNeasy total RNA kit (Qiagen, Hilden, Germany) for RNA preparation were employed in this research. Realtime quantitative- (qRT-) PCR utilized primers that were designed according to GenBank sequences and based on previous studies $[25,26]$. The primers were synthesized by Pacific Science Co., Ltd., Bangkok, Thailand. The human ELISA kit was purchased from BioLegend (San Diego, CA, USA). The other reagents used are described below.

2.2. Study Design, Study Population, and Ethical Considerations. The design of this study is summarized in Figure 1. This study comprised 55 male Thai citizens including 13 healthy volunteers (N group), 24 patients diagnosed with hyperlipidemia ( $\mathrm{H}$ group), and 18 patients with coronary heart disease (CHD group). The patients were examined and treated by a physician from Phramongkutklao Hospital.

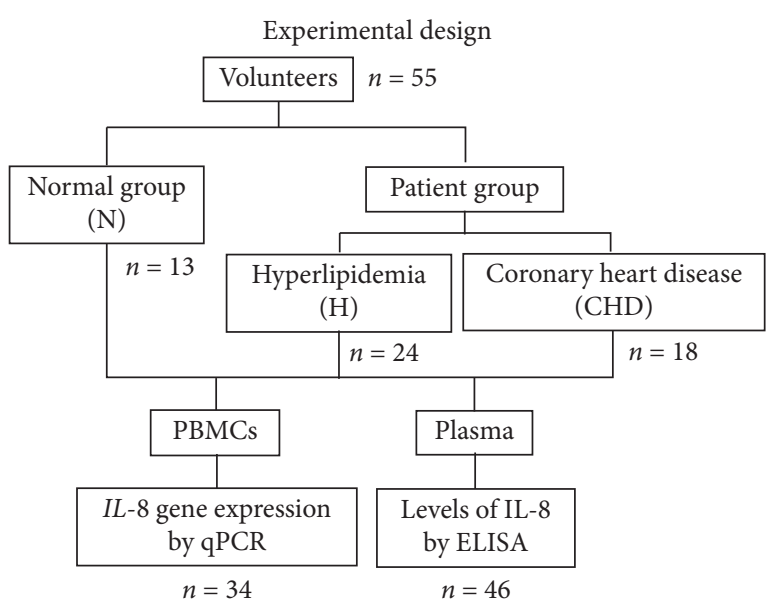

FIgURE 1: Experimental design.

They were classified based on their clinical manifestations according to the American College of Cardiology/American Heart Association criteria (2013) [27]. The H group had high cholesterol levels (total cholesterol (TC), LDL, and highdensity lipoprotein (HDL)), but it showed no evidence of vital organ dysfunction. Before inclusion, it was confirmed that the participants from the $\mathrm{N}$ and $\mathrm{H}$ groups had not received any cholesterol or blood pressure-lowering medication. The CHD patients were about to undergo coronary bypass grafting under the supervision of KP. All the CHD patients had been treated continuously with statin drugs since they were diagnosed and after surgery: 8 of 18 patients received $20 \mathrm{mg} /$ day of rosuvastatin and 10 of 18 patients received $40 \mathrm{mg} /$ day of atorvastatin. The $\mathrm{N}$ and $\mathrm{H}$ patient groups did not have any infections or underlying diseases. The $\mathrm{N}$ group had no cardiovascular risk factors.

This study was approved by the RSU ethical review board (RSUERB2018-001), the Faculty of Tropical Medicine, Mahidol University (MUTM 2012-031-01), and Phramongkutklao Hospital (Q004q/55_Exp). Before enrollment, all the participants were informed of the aims and benefits of the study before completing an informed consent form.

2.3. Blood Sample Collection. Five milliliter of heparinized blood was collected from each participant of the three groups. Two milliliter of plasma was stored at $-70^{\circ} \mathrm{C}$ for the detection of IL- 8 by ELISA. The packed blood cells were resuspended in D-PBS and used to isolate the peripheral mononuclear cells (PBMCs). These were separated by Isoprep gradient centrifugation (Robbins Scientific Corporation) as described previously [28]. Approximately 2 million PBMCs in TRIzol (Invitrogen ${ }^{\mathrm{TM}}$ ) were kept at $-70^{\circ} \mathrm{C}$ for the qRT-PCR.

2.4. Lipid Test. Lipid profiles including TC, triglyceride (TG), LDL cholesterol (LDL-c), and HDL cholesterol were determined by a biochemistry analyzer (Architect CI 16200, Abbott Laboratories, Abbott Park, IL, USA) at the clinical laboratory center of Phramongkutklao Hospital and/or by the Bangkok RIA Laboratory. 
2.5. qRT-PCR Analysis of IL8 mRNA Expression. The total RNA was isolated from the PBMCs using the RNeasy extraction kit (Qiagen) according to the manufacturer's instructions. The IL8 mRNA was amplified by qPCR using the SuperScript $^{\mathrm{TM}}$ III One-Step RT-PCR System with Platinum ${ }^{\mathrm{TM}}$ Taq DNA Polymerase according to the manufacturer's instructions (Invitrogen ${ }^{\mathrm{TM}}$, Carlsbad, CA, USA). Amplification was conducted in a Bio-Rad CFX Connect Real-Time System (Bio-Rad Laboratories, Inc., Hercules, CA, USA) using the following primers: cIL-8F (forward) 5'-GGCACAAACTTTCAGAGACAG-3' and cIL-8R (reverse) 5'-ACACAGAGCTGCAGAAATCAGG-3' [25]; glyceraldehyde 3phosphate dehydrogenase (GADPH) was used as a housekeeping gene to normalize the relative expression of IL8 with the primers: G6F (forward) 5'-ACAGAGTGAGCCCTTCTTCAA-3' and G6R (reverse) 5'-GGAGGCTGCATCATCGTACT- $3^{\prime}$ [26]. PCR conditions were $94^{\circ} \mathrm{C}$ for $2 \mathrm{~min}$ followed by 40 cycles of $95^{\circ} \mathrm{C}$ for $15 \mathrm{~s}, 60^{\circ} \mathrm{C}$ for $30 \mathrm{~s}$, and $76^{\circ} \mathrm{C}$ for $60 \mathrm{~s}$. Calculations of relative expression levels were performed using the $2^{-\Delta \Delta C t}$ method [28]. The qRT-PCR reactions were performed in quadruplicate.

2.6. Determination of Plasma IL-8 Levels Using ELISA. Levels of plasma IL-8 were determined by Human IL-8 ELISA MAX ${ }^{\text {тм }}$ Deluxe (BioLegend, San Diego, CA, USA) according to the manufacturer's recommendations. In brief, 96 wells were coated with a capture antibody and incubated overnight at $2-8^{\circ} \mathrm{C}$. Plasma samples $(100 \mu \mathrm{L})$ from the three groups and prediluted standards were added and incubated for $2 \mathrm{~h}$ at room temperature. Then, the wells were washed to remove any unbound material, and $100 \mu \mathrm{L}$ of diluted detection antibody was added for $60 \mathrm{~min}$ at room temperature. After the unbound materials were removed, $100 \mu \mathrm{L}$ of avidin-horseradish peroxidase conjugate was added for $30 \mathrm{~min}$ at room temperature. The bound enzyme was detected by adding $100 \mu \mathrm{L}$ of substrate solution $\mathrm{C}$ to each well and the reactions were stopped by adding $100 \mu \mathrm{L}$ of stop solution. The optical density was determined at $450 \mathrm{~nm}$ using the Tecan Sunrise OEM Remote Microplate Absorbance Reader (Tecan, Grödig, Austria). All the samples were assayed in triplicate. The IL-8 levels were calculated from standard curves.

2.7. Statistical Analysis. Clinical data were presented as medians (upper and lower range limits). The IL $8 \mathrm{mRNA}$ expression was represented as a fold change relative to the GADPH mRNA expression in the PBMCs. The IL-8 protein levels were expressed as a mean \pm SEM. The significant difference between two or more groups was determined by the Mann-Whitney $U$ test and the Kruskal-Wallis test, respectively. The correlations between CHD development/improvement and the IL8 mRNA expression or the plasma IL-8 levels were determined by Spearman's rho correlation analysis. Significance was set at $p<0.05$ at a $95 \%$ confidence interval. All statistical analyses were performed using SPSS software version 18 (SPSS, Chicago, IL, USA).

\section{Results}

3.1. Clinical Manifestations. The patients' characteristics, clinical manifestations, and the healthy controls were compared between groups and summarized, as shown in Table 1. There was no significant difference in age between the $\mathrm{N}$ and $\mathrm{H}$ groups $(p>0.05)$. The CHD patients were significantly older than the participants in the $\mathrm{N}$ and $\mathrm{H}$ groups. The levels of TC $(p<0.0001)$ and LDL $(p=0.0002)$ were significantly higher in the $\mathrm{H}$ group than in the $\mathrm{N}$ group, and the levels of "bad" lipids including TC ( $p=0.0028)$, TG $(p=0.0183)$, and LDL $(p=0.045)$ were significantly higher in the $\mathrm{H}$ group than in the $\mathrm{CHD}$ group. The $\mathrm{CHD}$ patients had a lipid baseline that was not significantly different when compared to $\mathrm{N}$ group participants, except for TG $(p=0.0387)$.

3.2. IL8 mRNA Expression. The IL8 mRNA expression levels are represented as relative expression (fold change) (Figure 2(a)). The IL8 mRNA expression in the CHD group was significantly lower than that in the $\mathrm{N}(p=0.0111)$ and $\mathrm{H}$ groups ( $p=0.0297$ ). The IL8 mRNA expression tended to be higher in the $\mathrm{H}$ group than in the $\mathrm{N}$ group $(p>0.05)$.

3.3. Levels of IL-8 in Plasma. The levels of plasma IL-8 in all the groups are shown in Figure 2(b). Similar to the IL8 pattern expression, the level of IL- 8 was higher in the $\mathrm{H}$ group compared to the $\mathrm{N}(p>0.05)$ and $\mathrm{CHD}(p=0.0210)$ groups. Levels did not differ significantly between the $\mathrm{N}$ and the CHD groups $(p>0.05)$.

3.4. Correlation between the IL-8 Expression and CHD Improvement. There was a significant negative correlation between IL8 mRNA $(r=-0.499, p=0.0248)$ or the plasma IL-8 level $(r=-3875, p=0.0195)$ and CHD improvement. The IL-8 and other parameters did not show significant correlation.

\section{Discussion}

This cross-sectional study used $\mathrm{N}, \mathrm{H}$, and $\mathrm{CHD}$ groups to illustrate atherogenesis development from healthy to atherosclerosis (moderate risk of CHD), consequent CHD, and the final improvement of plaques in coronary arteries. This is in accord with previous studies, which found that the reduction in the LDL-C level and the degree of anti-inflammation are inversely related to atherosclerotic progression $[29,30]$. We chose only male volunteers to control the influence of the sex-hormone factor such as estrogen. Because estrogens are primary examples of female sex steroids, the epidemiological studies in animal models have shown that estrogen has protective effects in cardiovascular disease. Previous evidence has also suggested that estrogen protects women against CHD premenopause (reviewed in [31]).

Although research into the functions of IL- 8 has proved controversial, most previous studies reporting the contribution of the IL- 8 expression to the progression of cardiovascular disorders involved both acute and chronic 
TABLE 1: General description and clinical manifestations of the study population and the comparison between groups.

\begin{tabular}{|c|c|c|c|c|c|c|}
\hline \multirow{2}{*}{ Variable } & $\begin{array}{l}\text { Normal } \\
(\mathrm{N})\end{array}$ & $\begin{array}{l}\text { Hyperlipidemia } \\
(\mathrm{H})\end{array}$ & $\begin{array}{c}\text { Coronary heart disease } \\
\text { (CHD) }\end{array}$ & \multicolumn{3}{|c|}{$p$ value } \\
\hline & $(n=13)$ & $(n=24)$ & $(n=18)$ & $\mathrm{N}$ vs. $\mathrm{H}$ & $\mathrm{H}$ vs. $\mathrm{CHD}$ & $\mathrm{N}$ vs. $\mathrm{CHD}$ \\
\hline Age (year) & $45.5(23-59)$ & $41(26-58)$ & $65.5(58-78)$ & 0.5577 & $<0.0001$ & $<0.0001$ \\
\hline $\mathrm{TC}(\mathrm{mg} / \mathrm{dL})$ & $178.5(150-199)$ & $226.5(200-344)$ & $171(115-259)$ & $<0.0001$ & 0.0028 & 0.6548 \\
\hline $\mathrm{TG}(\mathrm{mg} / \mathrm{dL})$ & $164.5(76-242)$ & $172(70-280)$ & $84(65-169)$ & 0.9157 & 0.0183 & 0.0387 \\
\hline $\mathrm{HDL}(\mathrm{mg} / \mathrm{dL})$ & $42.5(26-60)$ & $45(30-80)$ & $49(39-75)$ & 0.5276 & 0.2624 & 0.1576 \\
\hline $\mathrm{LDL}(\mathrm{mg} / \mathrm{dL})$ & $113(63-124)$ & $145(70-190)$ & $90(44-174)$ & 0.0002 & 0.045 & 0.6932 \\
\hline
\end{tabular}

All normal controls and patients were male. $\mathrm{N}=$ normal controls; $\mathrm{H}$ and $\mathrm{CHD}=$ patients with hyperlipidemia and coronary heart disease, respectively. $\mathrm{TC}=$ total cholesterol; $\mathrm{TG}=$ triglyceride; $\mathrm{HDL}=$ high-density lipoprotein; $\mathrm{LDL}=$ low-density lipoprotein. Data are shown as median (ranges). The differences in each variable between two groups ( $\mathrm{N}$ vs. $\mathrm{H}, \mathrm{H}$ vs. $\mathrm{CHD}$, and $\mathrm{N}$ vs. $\mathrm{CHD}$ ) were determined using the Mann-Whitney $U$ test. The $\alpha$ level was set at $<0.05$ at a $95 \%$ confidence interval.

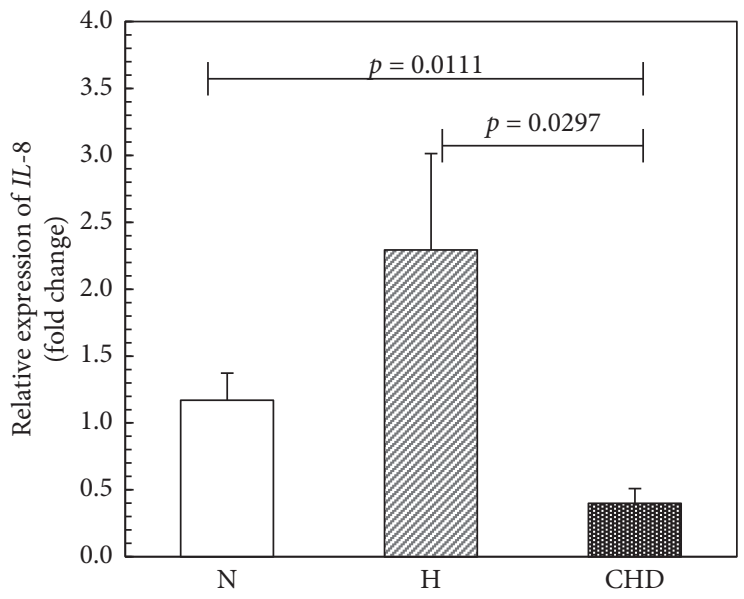

(a)

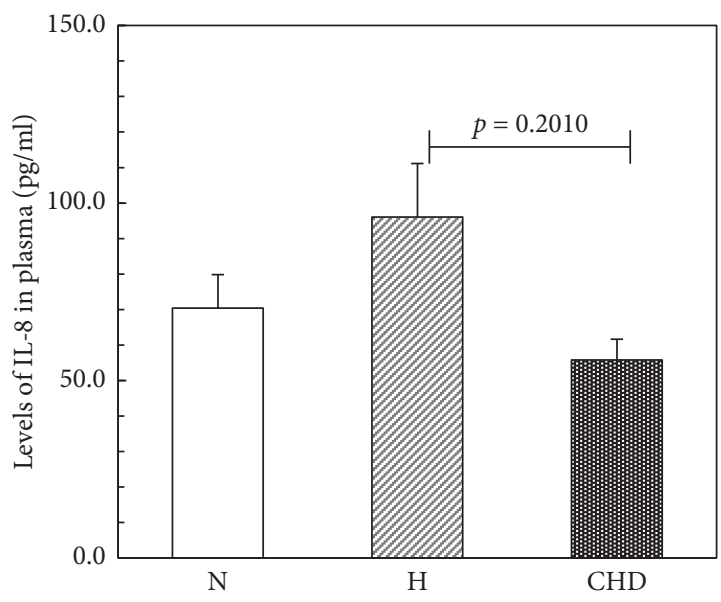

(b)

FIGURE 2: IL-8 expression and its association to clinical manifestation. (a) IL8 mRNA expression (2.0-fold change) relative to GADPH mRNA in peripheral blood mononuclear cells obtained from normal controls $\mathrm{N}$, hyperlipidemia $\mathrm{H}$, and patients with acute coronary heart disease (CHD), as determined by qRT-PCR. Calculations of relative expression levels were performed using the 2- ${ }^{\Delta \Delta C t}$ method [26]. qRTPCR reactions were performed in quadruplicate. (b) Plasma levels (pg/ml) of IL-8 from N, H, and CHD groups using ELISA. The assay was performed in quadruplicate. Data are presented as mean \pm SEM.

vascular inflammation [17-20]. IL-8 is a cytokine that is released in high amounts from neutrophils that participate in all stages of atherosclerosis and the development of CHD [17-19]. IL-8 is mediated through various mechanisms that contribute to cardiovascular disorders [12-20, 32-36]. IL-8 from neutrophils was found in both the circulation and the intraplaque of carotid stenosis. This finding indicates that IL-8 is critical for plaque development and progression [37]. In atherogenesis, IL-8 is a chemoattractant [36] that promotes monocyte adherence to the vascular endothelium [33] and has mitogenic effects on vascular smooth muscle cells [36]. IL-8 controls the cholesterol efflux via upregulating miR-183 in macrophage-derived foam cells [33]. IL-8 is also involved in the migration of monocytes into the subendothelial space which is a crucial process in the early stages of atherosclerosis $[32,38]$. IL-8 is mediated through the downregulation of the tissue inhibitor metalloproteinase- 1 to contribute atherogenesis (reviewed in $[26,34])$. Moreover, the polymorphism of IL-8-251A/T contributes to CHD susceptibility in Chinese populations $[20,21]$.

In acute inflammation, IL-8 functions in chemotaxis and induces migration of neutrophils and macrophages to the tunica intima of the subendothelial space, sites of injury, or endothelial fatty streaks [17]. Localization of monocyte chemoattractant protein-1 (MCP-1) and IL-8 is found in human atheroma. An earlier in vitro study proved that IL-8 was a powerful trigger for firm adhesion of monocytes to the vascular endothelium under flow conditions [33]. In addition, elevated baseline plasma levels of IL- 8 have been associated with an increased risk of long-term all-cause mortality in patients with acute coronary syndrome. Furthermore, this association was independent of a variety of clinical, laboratory, and angiographic variables including contemporary biomarkers with established prognostic efficacy in acute coronary syndrome [10].

Statins are selective competitive inhibitors of 3-hydroxy3-methyl-glutaryl-CoA reductase that interfere with 
mevalonate-derived compounds and lower cholesterol. Therefore, statins are used to treat hypercholesterolemia and are involved in the primary and secondary prevention of cardiovascular diseases. Rosuvastatin and atorvastatin are newly developed statins, and the former can lower LDL-C more effectively than the latter (reviewed in [39]). This study showed that CHD patients taking $20 \mathrm{mg}$ of rosuvastatin or $40 \mathrm{mg}$ of atorvastatin achieved the LDL-C goal $(<100 \mathrm{mg} / \mathrm{dl})$. These findings were in line with previous 30-day treatment studies $[40,41]$. Also, recent research has revealed that the LDL-C goal was not time-dependent [42].

Several previous studies found that statin drugs have played a role in the anti-inflammation of cardiovascular disorders $[39,43,44]$. Atorvastatin was shown to attenuate neutrophil migration after two weeks of exposure to a high dose (80 mg) and to decrease the IL- 8 expression in a dose-dependent manner [43]. In addition, statins inhibit leukocyte and endothelial cell interactions and reduce inflammatory cell numbers within atherosclerotic plaques (reviewed in [39]. Prior research has suggested that rosuvastatin suppresses the inflammatory response through a mechanism involving the inhibition of c-Jun N-terminal kinase and NF- $\kappa \mathrm{B}$. Moreover, it has been found to reduce adhesion molecules and some cytokines including IL-8, IL-6, and MCP-1 [44]. Due to the ability of the statin group to reduce inflammatory cytokine production, statin therapy has been applied as an alternative in some infectious diseases to prevent overuse-related antibiotic resistance, e.g., in intestinal Salmonella infections [45], Pseudomonas pneumonia [46], and chronic obstructive pulmonary disease (COPD) [47].

The therapeutic effects of rosuvastatin and atorvastatin reduce bad lipids, decrease vascular inflammation, and result in the regression of coronary atherosclerotic plaques [48]. In this meta-analysis study, intravascular ultrasound imaging demonstrated that both drugs could significantly reduce atheroma volume, increase the lumen volume of the coronary artery, and regress the composition of atherosclerotic plaques. Regression and stabilization of plaque prevent rupture that leads to vascular occlusion.

In our cross-sectional study, we found that the IL 8 mRNA expression and the IL-8 plasma protein levels increased concordantly in the $\mathrm{H}$ group compared to healthy control. In contrast, we observed a significant decrease in the IL8 mRNA expression $(p=0.0126)$ and the corresponding protein levels $(p=0.0247)$ in CHD patients compared with the $\mathrm{H}$ group participants. We assume that the decrease in the IL-8 expression in the CHD group was caused by the action of cholesterol-lowering medications, as previously described by patients taking rosuvastatin and atorvastatin [44]. These results concur with Yeh et al. who recently reported a lower CAD risk among all statin users that was not duration dependent [42].

A previous study has demonstrated that IL-18 was a potential predictive marker for atherosclerosis progression in SIV-infected rhesus monkeys (Macaca mulatta) on a high-fat/high-cholesterol diet [25]. Similarly, we suppose that the reduced IL- 8 expression among the statin-grouptreated patients for CHD might illustrate the chronic inflammation regression of coronary arteries and other vasculature. Even though the regression of atherosclerotic plaque was not ultrasonically tested, we assumed that after treatment with rosuvastatin and atorvastatin, the pathologic changes of plaque in the coronary arteries were signs of recovery. Prior research agrees with our hypothesis that statin treatment not only decreases LDL but also results in plaque improvement $[48,49]$.

This study has several limitations. First, the study did not include CHD patients who had never been treated with statins or other cholesterol-lowering drugs. We lack some information from the patients' characteristics such as the detail of coronary graft bypass or clinical history and the progress of disease. However, reliable literature has mostly reported an increase in the IL8 mRNA expression and IL-8 levels in cardiovascular diseases and coronary artery syndrome. IL-8 is mediated through various mechanisms to contribute to cardiovascular disorders [12-20, 32-36]. Second, the size of the study population was small and not designed using a priori calculations of statistical power. Therefore, these findings need to be confirmed with larger sample sizes in further studies. Third, in this study, we did not determine the pathologic change in the atherosclerosis plaques in the CHD patient group. Therefore, we could not confirm the improvement of plaque in the vessels after treatment although previous studies suggest that the therapeutic effects of rosuvastatin and atorvastatin result in the regression of coronary atherosclerotic plaques [48]. Fourth, other pivotal cytokines, such as TNF- $\alpha$, MCP-1, IL- $1 \beta$, and IL-6, should be considered for future research [2].

\section{Conclusion}

This study found that both the transcriptomic and the phenotypic IL-8 expression increased in hyperlipidemia. In contrast, a significant decrease in the amount of IL- 8 was observed in Thai CHD patients who took statin medication. Based on our findings and previous studies on the role of IL8 in the pathogenesis of CVD, IL- 8 may have the potential to be used as an alternative marker to evaluate the therapeutic effects of the statin drug group.

\section{Data Availability}

The data used to support the findings of this study are available from the corresponding author upon request.

\section{Conflicts of Interest}

The authors declare that they have no conflicts of interest.

\section{Acknowledgments}

This study was supported by a grant from Rangsit University (grant no. 45/2560), the Faculty of Tropical Medicine, Mahidol University, and the Mahidol University Research Fund of the National Research Council of Thailand (20112012). The authors thank the volunteers and patients who donated their blood and the staff at Phramongkutklao Hospital for their cooperation in blood collection, taking consent from patients, and filling out the questionnaire voluntarily. 


\section{References}

[1] C. Weber and H. Noels, "Atherosclerosis: current pathogenesis and therapeutic options," Nature Medicine, vol. 17, no. 11, pp. 1410-1422, 2011.

[2] P. Libby, "Inflammation in atherosclerosis," Arteriosclerosis, Thrombosis, and Vascular Biology, vol. 32, no. 9, pp. 20452051, 2012.

[3] A. M. Carter, "Complement activation: an emerging player in the pathogenesis of cardiovascular disease," Scientifica, vol. 2012, Article ID 402783, 14 pages, 2012.

[4] D. P. Ramji and T. S. Davies, "Cytokines in atherosclerosis: key players in all stages of disease and promising therapeutic targets," Cytokine \& Growth Factor Reviews, vol. 26, no. 6, pp. 673-685, 2015.

[5] C. A. Dinarello, "Interleukin-1 in the pathogenesis and treatment of inflammatory diseases," Blood, vol. 117, no. 14, pp. 3720-3732, 2011.

[6] E. Zakynthinos and N. Pappa, "Inflammatory biomarkers in coronary artery disease," Journal of Cardiology, vol. 53, no. 3, pp. 317-333, 2009.

[7] J. Van Damme and G. Opdenakker, "Interaction of interferons with skin reactive cytokines: from interleukin-1 to interleukin-8," Journal of Investigative Dermatology, vol. 95, no. 6, pp. 90S-93S, 1990.

[8] D.-H. Kim, B.-Y. Rhim, S.-K. Eo, and K. Kim, "Differential regulation of CC chemokine ligand 2 and CXCL8 by antifungal agent nystatin in macrophages," Biochemical and Biophysical Research Communications, vol. 437, no. 3, pp. 392-396, 2013.

[9] M. Takami, V. Terry, and L. Petruzzelli, "Signaling pathways involved in IL-8-dependent activation of adhesion through Mac-1," The Journal of Immunology, vol. 168, no. 9, pp. 4559-4566, 2002.

[10] E. Cavusoglu, J. D. Marmur, S. Yanamadala et al., "Elevated baseline plasma IL-8 levels are an independent predictor of long-term all-cause mortality in patients with acute coronary syndrome," Atherosclerosis, vol. 242, no. 2, pp. 589-594, 2015.

[11] I. M. Velásquez, P. Frumento, K. Johansson et al., "Association of interleukin 8 with myocardial infarction: results from the stockholm heart epidemiology program," International Journal of Cardiology, vol. 172, no. 1, pp. 173-178, 2014.

[12] Z. L. Zhang, W. C. Wu, J. Q. Liu et al., "Screening of differentially expressed genes related to ischemic stroke and functional analysis with DNA microarray," European Review for Medical and Pharmacological Sciences, vol. 18, no. 8, pp. 1181-1188, 2014.

[13] V. F. Azevedo, J. R. Faria-Neto, A. Stinghen et al., "IL-8 but not other biomarkers of endothelial damage is associated with disease activity in patients with ankylosing spondylitis without treatment with anti-TNF agents," Rheumatology International, vol. 33, no. 7, pp. 1779-1783, 2013.

[14] S. van Eijl, E. Mortaz, A. F. Ferreira et al., "Humic acid enhances cigarette smoke-induced lung emphysema in mice and IL-8 release of human monocytes," Pulmonary Pharmacology \& Therapeutics, vol. 24, no. 6, pp. 682-689, 2011.

[15] Y. Fujita, M. Nakayama, M. Naito et al., "Hemoglobin receptor protein from porphyromonas gingivalis induces interleukin-8 production in human gingival epithelial cells through stimulation of the mitogen-activated protein kinase and NF- $\kappa \mathrm{B}$ signal transduction pathways," Infection and Immunity, vol. 82, no. 1, pp. 202-211, 2014.

[16] T. Yoshio, H. Okamoto, K. Kurasawa, Y. Dei, S. Hirohata, and S. Minota, "IL-6, IL-8, IP-10, MCP-1 and G-CSF are significantly increased in cerebrospinal fluid but not in sera of patients with central neuropsychiatric lupus erythematosus," Lupus, vol. 25, no. 9, pp. 997-1003, 2016.

[17] S. M. Boekholdt, R. J. G. Peters, C. E. Hack et al., "IL-8 plasma concentrations and the risk of future coronary artery disease in apparently healthy men and women," Arteriosclerosis, Thrombosis, and Vascular Biology, vol. 24, no. 8, pp. 1503-1508, 2004.

[18] T. Inoue, H. Komoda, M. Nonaka, M. Kameda, T. Uchida, and K. Node, "Interleukin-8 as an independent predictor of longterm clinical outcome in patients with coronary artery disease," International Journal of Cardiology, vol. 124, no. 3, pp. 319-325, 2008.

[19] E. Romuk, B. Skrzep-Poloczek, C. Wojciechowska et al., "Selectin-P and interleukin-8 plasma levels in coronary heart disease patients," European Journal of Clinical Investigation, vol. 32, no. 9, pp. 657-661, 2002.

[20] R. J. Zhang, X. D. Li, S. W. Zhang, X. H. Li, and L. Wu, "IL-8$251 \mathrm{~A} / \mathrm{T}$ polymorphism contributes to coronary artery disease susceptibility in a Chinese population," Genetics and Molecular Research, vol. 16, no. 1, 2017.

[21] N. G. Frangogiannis, "Chemokines in the ischemic myocardium: from inflammation to fibrosis," Inflammation Research, vol. 53, no. 11, pp. 585-595, 2004.

[22] K. Schömig, G. Busch, B. Steppich et al., "Interleukin-8 is associated with circulating CD133+ progenitor cells in acute myocardial infarction," European Heart Journal, vol. 27, no. 9, pp. 1032-1037, 2006.

[23] X. Zhao, W. Zhang, D. Xing et al., "Endothelial cells overexpressing IL-8 receptor reduce cardiac remodeling and dysfunction following myocardial infarction," American Journal of Physiology-Heart and Circulatory Physiology, vol. 305, no. 4, pp. H590-H598, 2013.

[24] J. H. Yearley, D. Xia, C. B. Pearson, A. Carville, R. P. Shannon, and K. G. Mansfield, "Interleukin-18 predicts atherosclerosis progression in SIV-infected and uninfected rhesus monkeys (Macaca mulatta) on a high-fat/high-cholesterol diet," Laboratory Investigation, vol. 89, no. 6, pp. 657-667, 2009.

[25] T. Angrisano, R. Pero, S. Peluso et al., "LPS-induced IL-8 activation in human intestinal epithelial cells is accompanied by specific histone $\mathrm{H} 3$ acetylation and methylation changes," BMC Microbiology, vol. 10, no. 1, p. 172, 2010.

[26] T. Sophonnithiprasert, S. Nilwarangkoon, Y. Nakamura, and R. Watanapokasin, "Goniothalamin enhances TRAIL-induced apoptosis in colorectal cancer cells through DR5 upregulation and cFLIP downregulation," International Journal of Oncology, vol. 47, no. 6, pp. 2188-2196, 2015.

[27] D. C. Goff Jr., D. M. Lloyd-Jones, G. Bennett et al., "2013 ACC/AHA guideline on the assessment of cardiovascular risk: a report of the American college of cardiology/American heart association task force on practice guidelines," Journal of the American College of Cardiology, vol. 63, pp. 2935-2959, 2014.

[28] W. Dechkajorn, S. Benjathummarak, R. Kumsiri, and Y. Maneerat, "The role of the BAFF/APRIL system in the T cell-independent specific response to blood stage Plasmodium falciparum hemozoin," Cytokine, vol. 111, pp. 445-453, 2018.

[29] R. Puri, S. E. Nissen, P. Libby et al., "C-reactive protein, but not low-density lipoprotein cholesterol levels, associate with coronary atheroma regression and cardiovascular events after maximally intensive statin therapy," Circulation, vol. 128, no. 22, pp. 2395-2403, 2013.

[30] R. Puri, S. E. Nissen, M. Shao et al., "Impact of baseline lipoprotein and C-reactive protein levels on coronary atheroma regression following high-intensity statin therapy," The 
American Journal of Cardiology, vol. 114, no. 10, pp. 14651472, 2014.

[31] J.-F. Arnal, P.-Y. Scarabin, F. Trémollières, H. Laurell, and P. Gourdy, "Estrogens in vascular biology and disease: where do we stand today?" Current Opinion in Lipidology, vol. 18, no. 5, pp. 554-560, 2007.

[32] S. Apostolakis, E. G. Papadakis, E. Krambovitis, and D. A Spandidos, "Chemokines in vascular pathology (review)," International Journal of Molecular Medicine, vol. 17, no. 5, pp. 691-701, 2006.

[33] R. E. Gerszten, E. A. Garcia-Zepeda, Y.-C. Lim et al., "MCP-1 and IL-8 trigger firm adhesion of monocytes to vascular endothelium under flow conditions," Nature, vol. 398, no. 6729 , pp. 718-723, 1999.

[34] W. S. Shin, A. Szuba, and S. G. Rockson, "The role of chemokines in human cardiovascular pathology: enhanced biological insights," Atherosclerosis, vol. 160, no. 1, pp. 91-102, 2002.

[35] X. E. Tang, H. Li, L. Y. Chen et al., "IL-8 negatively regulates ABCA1 expression and cholesterol efflux via upregulating miR-183 in THP-1 macrophage-derived foam cells," Cytokine, vol. 122, Article ID 154385, 2019.

[36] T. L. Yue, X. Wang, C. P. Sung et al., "Interleukin-8. A mitogen and chemoattractant for vascular smooth muscle cells," Circulation Research, vol. 75, no. 1, pp. 1-7, 1994.

[37] F. Marino, A. M. Maresca, L. Castiglioni et al., "Simvastatin down-regulates the production of interleukin- 8 by neutrophil leukocytes from dyslipidemic patients," BMC Cardiovascular Disorders, vol. 14, no. 1, p. 37, 2014.

[38] R. E. Gerszten, J. Chen, M. Ishli et al., "Specificity of the thrombin receptor for agonist peptide is defined by its extracellular surface," Nature, vol. 368, no. 6472, pp. 648-651, 1994.

[39] J. K. Liao, "Beyond lipid lowering: the role of statins in vascular protection," International Journal of Cardiology, vol. 86, no. 1, pp. 5-18, 2002.

[40] J. M. McKenney, P. H. Jones, M. A. Adamczyk, V. A. Cain, B. S. Bryzinski, and J. W. Blasetto, "Comparison of the efficacy of rosuvastatin versus atorvastatin, simvastatin, and pravastatin in achieving lipid goals: results from the STELLAR trial," Current Medical Research and Opinion, vol. 19, no. 8, pp. 689-698, 2003.

[41] F. Pelliccia, G. Rosano, G. Marazzi et al., "Pharmacodynamic effects of atorvastatin versus rosuvastatin in coronary artery disease patients with normal platelet reactivity while on dual antiplatelet therapy-the PEARL randomized cross-over study," European Journal of Pharmacology, vol. 725, pp. 1822, 2014.

[42] J.-J. Yeh, C.-L. Lin, C. Y. Hsu, Z. Shae, and C.-H. Kao, "Associations between statins and coronary artery disease and stroke risks in patients with asthma-chronic obstructive pulmonary disease overlap syndrome: a time-dependent regression study," Atherosclerosis, vol. 283, pp. 61-68, 2019.

[43] Y. R. Chee, R. W. Watson, J. McCarthy et al., "High dose statin prophylaxis in cardiopulmonary bypass related surgery: clinical utility," Journal of Cardiothoracic Surgery, vol. 12, no. 1, p. 20, 2017.

[44] D. F. Gualtero, S. M. Viafara-Garcia, S. J. Morantes, D. M. Buitrago, O. A. Gonzalez, and G. I. Lafaurie, "Rosuvastatin inhibits interleukin (IL)-8 and IL-6 production in human coronary artery endothelial cells stimulated with aggregatibacter actinomycetemcomitans serotype b," Journal of Periodontology, vol. 88, no. 2, pp. 225-235, 2017.
[45] F. C. Huang and S. C. Huang, "Differential effects of statins on inflammatory interleukin-8 and antimicrobial peptide human beta-defensin 2 responses in salmonella-infected intestinal epithelial cells," International Journal of Molecular Sciences, vol. 19, no. 6, 2018.

[46] A. Iwata, R. Shirai, H. Ishii et al., "Inhibitory effect of statins on inflammatory cytokine production from human bronchial epithelial cells," Clinical \& Experimental Immunology, vol. 168, no. 2, pp. 234-240, 2012.

[47] M. Sanja, P. Jozsef, P. G. Sanja et al., "Cytokines and statin therapy in chronic obstructive pulmonary disease patients," Scandinavian Journal of Clinical and Laboratory Investigation, vol. 78, no. 7-8, pp. 533-538, 2018.

[48] C. Qian, B. Wei, J. Ding et al., "Meta-analysis comparing the effects of rosuvastatin versus atorvastatin on regression of coronary atherosclerotic plaques," The American Journal of Cardiology, vol. 116, no. 10, pp. 1521-1526, 2015.

[49] A. M. Noyes and P. D. Thompson, "A systematic review of the time course of atherosclerotic plaque regression," Atherosclerosis, vol. 234, no. 1, pp. 75-84, 2014. 